\title{
Multiplicative Jordan derivations on triangular n-matrix rings
}

\author{
Huimin Chen, Xiaofei Qi* \\ School of Mathematical Science, Shanxi University, Taiyuan 030006 China \\ *Corresponding author, e-mail: xiaofeiqisxu@aliyun.com
}

Received 24 Jul 2020

Accepted 21 Oct 2020

\begin{abstract}
Let $\mathscr{T}$ be a triangular $n$-matrix ring $(n \geqslant 2)$ and $\delta: \mathscr{T} \rightarrow \mathscr{T}$ a map. It is shown that $\delta$ is a multiplicative Jordan derivation if and only if one of the statements holds: (1) if $\mathscr{T}$ is 2-torsion free, then $\delta$ is an additive derivation; (2) if $\mathscr{T}$ is 2-torsion, under some mild assumptions, then $\delta(X)=d(X)+\gamma(X)$ holds for all $X \in \mathscr{T}$, where $d: \mathscr{T} \rightarrow \mathscr{T}$ is an additive derivation and $\gamma$ is a map from $\mathscr{T}$ into its center vanishing on all elements $X Y+Y X$ for $X, Y \in \mathscr{T}$. This generalizes some related known results.
\end{abstract}

KEYWORDS: Jordan derivations, derivations, triangular rings, triangular $n$-matrix rings

MSC2010: 16W10 47B47

\section{INTRODUCTION}

Let $\mathscr{A}$ be an associative ring or an algebra. For any $A, B \in \mathscr{A}$, the Jordan product of $A, B$ is defined by $A \circ B=A B+B A$. Recall that a map $\delta$ : $\mathscr{A} \rightarrow \mathscr{A}$ is called a multiplicative (or nonlinear) derivation if $\delta(A B)=\delta(A) B+A \delta(B)$ for all $A, B \in$ $\mathscr{A}$; is called a multiplicative (or nonlinear) Jordan derivation if $\delta(A \circ B)=\delta(A) \circ B+A \circ \delta(B)$ for all $A, B \in \mathscr{A}$. Particularly, if $\delta$ is also assumed to be additive (linear), then $\delta$ is called additive (linear) derivation and Jordan derivation, respectively. The questions of characterizing (multiplicative) Jordan derivations and revealing the relationship between Jordan derivations and derivations have received many mathematicians' attention (for example, see [1-7] and the references therein).

In [8], the author introduced a class of rings (or algebras) as follows. Let $\mathscr{A}$ and $\mathscr{B}$ be unital rings (or algebras over a commutative ring $\mathscr{R}$ ), and $\mathscr{M}$ an $(\mathscr{A}, \mathscr{B})$-bimodule which is faithful as a left $\mathscr{A}$-module as well as a right $\mathscr{B}$-module. The associative ring (or $\mathscr{R}$-algebra)

$\operatorname{Tri}(\mathscr{A}, \mathscr{M}, \mathscr{B})=\left\{\left(\begin{array}{cc}A & M \\ 0 & B\end{array}\right) \mid A \in \mathscr{A}, M \in \mathscr{M}, B \in \mathscr{B}\right\}$

under the usual matrix operations is called a triangular ring (or algebra). Zhang and Yu [7] show that every linear Jordan derivation on a triangular algebra $\mathscr{U}$ is a derivation under the assumption that the commutative ring $\mathscr{R}$ is 2 -torsion free. Recall that a ring $\mathscr{A}$ is said to be 2 -torsion free if $2 a=0$ implies $a=0$ for any $a \in \mathscr{A}$; otherwise, $\mathscr{A}$ is 2 -torsion. Xiao [6] generalizes the result of [7] to multiplicative Jordan derivations and obtains the same result.

Recently, Ferreira [9] defined a class of ring called triangular $n$-matrix ring as follows.

Definition 1 ([9]) Let $\mathscr{R}_{1}, \mathscr{R}_{2}, \ldots, \mathscr{R}_{n}$ be unital rings and $\mathscr{M}_{i j}$ be $\left(\mathscr{R}_{i}, \mathscr{R}_{j}\right)$-bimodules with $\mathscr{M}_{i i}=\mathscr{R}_{i}$ for all $1 \leqslant i \leqslant j \leqslant n$. Let $\varphi_{i j k}: \mathscr{M}_{i j} \otimes_{\mathscr{R}_{j}} \mathscr{M}_{j k} \rightarrow \mathscr{M}_{i k}$ be $\left(\mathscr{R}_{i}, \mathscr{R}_{k}\right)$-bimodules homomorphisms with $\varphi_{i i j}$ : $\mathscr{R}_{i} \otimes_{\mathscr{R}_{i}} \mathscr{M}_{i j} \rightarrow \mathscr{M}_{i j}$ and $\varphi_{i j j}: \mathscr{M}_{i j} \otimes_{\mathscr{R}_{j}} \mathscr{R}_{j} \rightarrow \mathscr{M}_{i j}$ the canonical multiplication maps for all $1 \leqslant i \leqslant$ $j \leqslant k \leqslant n$. Write $a b=\varphi_{i j k}(a \otimes b)$ for all $a \in \mathscr{M}_{i j}$ and $b \in \mathscr{M}_{j k}$. Assume that $\mathscr{M}_{i j}$ is faithful as a left $\mathscr{R}_{i}$-module and faithful as a right $\mathscr{R}_{j}$-module for all $1 \leqslant i<j \leqslant n$. Let $\mathscr{T}=\mathscr{T}_{n}\left(\mathscr{R}_{i} ; \mathscr{M}_{i j}\right)$ be the set

$$
\mathscr{T}=\left\{\left(\begin{array}{ccccc}
r_{11} & m_{12} & \cdots & m_{1(n-1)} & m_{1 n} \\
0 & r_{22} & \cdots & m_{2(n-1)} & m_{2 n} \\
\vdots & \vdots & \ddots & \vdots & \vdots \\
0 & 0 & \cdots & r_{(n-1)(n-1)} & m_{(n-1) n} \\
0 & 0 & \cdots & 0 & r_{n n}
\end{array}\right) \mid\right.
$$

Furthermore, assume that $a(b c)=(a b) c$ for all $a \in$ $\mathscr{M}_{i k}, b \in \mathscr{M}_{k l}$, and $c \in \mathscr{M}_{l j}$ with $1 \leqslant i \leqslant k \leqslant l \leqslant$ $j \leqslant m$. Then, with the usual matrix operations, $\mathscr{T}$ is called a triangular $n$-matrix ring.

It is obvious that upper triangular matrix rings $\mathscr{T}_{n}(\mathscr{R})$ with $n \geqslant 3$ over a unital associative ring $\mathscr{R}$ 
are triangular $n$-matrix rings; and that triangular 2matrix rings are usual triangular rings. However, for $n \geqslant 3$, a triangular ring may not be a triangular $n$ matrix ring; conversely, a triangular $n$-matrix ring may not be a triangular ring (see Example 2.1 in $[10])$. For some results on triangular $n$-matrix rings, see $[9,11]$.

The purpose of the present paper is to discuss the structure of multiplicative Jordan derivations on triangular $n$-matrix rings for any $n \geqslant 2$. Assume that $\delta$ is a map on a triangular $n$-matrix ring $\mathscr{T}$. We show that $\delta$ is a multiplicative Jordan derivation if and only if one of the following statements is true: (1) if $\mathscr{T}$ is 2 -torsion free, then $\delta$ is an additive derivation; (2) if $\mathscr{T}$ is 2-torsion, then $\delta$ has the form $\delta(X)=d(X)+\gamma(X)$ for all $X \in \mathscr{T}$ under some mild assumptions on $\mathscr{T}$, where $d$ is an additive derivation on $\mathscr{T}$, and $\gamma$ is a map from $\mathscr{T}$ into its center vanishing on all elements $X Y+Y X$ for $X, Y \in$ T.

\section{MAIN RESULT}

For 2-torsion free triangular $n$-matrix rings, we have the following result.

Theorem 1 Let $\mathscr{T}$ be a 2-torsion free triangular $n$ matrix ring $(n \geqslant 2)$. Then a map $\delta: \mathscr{T} \rightarrow \mathscr{T}$ is a multiplicative Jordan derivation if and only if $\delta$ is an additive derivation.

Note that, if a ring $\mathscr{R}$ is 2 -torsion, then for any elements $T, S \in \mathscr{R}$, it is clear that $T S+S T=T S-S T$. In this case, Jordan derivations and Lie derivations are equivalent. Thus, by Theorem 2.3 in [12], we can give the characterization of multiplicative Jordan derivations on 2-torsion triangular $n$-matrix rings.

Fix any $i \in\{1,2, \ldots, n\}$. Let $E_{i}$ stand for the nontrivial idempotent in $\mathscr{T}$ whose elements with $(i, i)$ position 1 and the rest 0 . Write $P_{i}=E_{1}+E_{2}+$ $\cdots+E_{i}$ and $Q_{i}=I-P_{i}$.

Theorem 2 Let $\mathscr{T}$ be a 2-torsion triangular $n$ matrix ring $(n \geqslant 2)$. Assume that $P_{\left[\frac{n}{2}\right]} \mathscr{Z}(\mathscr{T}) P_{\left[\frac{n}{2}\right]}=$ $\mathscr{Z}\left(P_{\left[\frac{n}{2}\right]} \mathscr{T} P_{\left[\frac{n}{2}\right]}\right)$ and $Q_{\left[\frac{n}{2}\right]} \mathscr{Z}(\mathscr{T}) Q_{\left[\frac{n}{2}\right]}=\mathscr{Z}\left(Q_{\left[\frac{n}{2}\right]} \mathscr{T} Q_{\left[\frac{n}{2}\right]}\right)$. Then a map $\delta: \mathscr{T} \rightarrow \mathscr{T}$ is a multiplicative Jordan derivation if and only if $\delta(X)=d(X)+\gamma(X)$ holds for all $X \in \mathscr{T}$, where $d: \mathscr{T} \rightarrow \mathscr{T}$ is an additive derivation and $\gamma: \mathscr{T} \rightarrow \mathscr{Z}(\mathscr{T})$ is a map vanishing on any $[X, Y]=X Y+Y X$. Here, $[s]$ is the integer part of $s$ and $\mathscr{Z}(\mathscr{T})$ is the center of $\mathscr{T}$.

Particularly, if $n=2$, we can obtain a complete characterization of multiplicative Jordan deriva- tions on triangular rings, which is a generalization of the related results in $[6,7]$.

Corollary 1 Let $\mathscr{A}$ and $\mathscr{B}$ be unital rings, and $\mathscr{M}$ be an $(\mathscr{A}, \mathscr{B})$-bimodule, which is faithful as a left $\mathscr{A}$-module and also as a right $\mathscr{B}$-module. Let $\mathscr{U}=$ $\operatorname{Tri}(\mathscr{A}, \mathscr{M}, \mathscr{B})$ be the triangular ring. Then a map $\delta: \mathscr{U} \rightarrow \mathscr{U}$ is a multiplicative Jordan derivation if and only if the following statements hold:

(i) if $\mathscr{U}$ is 2-torsion free, then $\delta$ is an additive derivation;

(ii) if $\mathscr{U}$ is 2-torsion, $P \mathscr{Z}(\mathscr{U}) P=\mathscr{Z}(P \mathscr{U} P)$ and ( $I-$ $P) \mathscr{Z}(\mathscr{U})(I-P)=\mathscr{Z}((I-P) \mathscr{U}(I-P))$, then $\delta=$ $d+\gamma$, where $d: \mathscr{U} \rightarrow \mathscr{U}$ is an additive derivation and $\gamma: \mathscr{U} \rightarrow \mathscr{Z}(\mathscr{U})$ is a map vanishing on all commutators.

Set for $1 \leqslant i \leqslant j \leqslant n$,

$\mathscr{T}_{i j}=\left\{\left(m_{k t}\right) \mid m_{k t}=\left\{\begin{array}{ll}m_{i j}, & \text { if }(k, t)=(i, j), \\ 0, & \text { if }(k, t) \neq(i, j),\end{array}\right\} \subset \mathscr{T}\right.$.

Then we can write $\mathscr{T}=\sum_{1 \leqslant i \leqslant j \leqslant n} \mathscr{T}_{i j}$. Obviously, for any element $a_{i j} \in \mathscr{T}_{i j}, a_{i j} a_{k j}=0$ whenever $j \neq k$.

Denote by $\mathscr{A}_{i}=P_{i} \mathscr{T} P_{i}, \mathscr{B}_{i}=Q_{i} \mathscr{T} Q_{i}$ and $\mathscr{M}_{i}=$ $P_{i} \mathscr{T} Q_{i}(i \in\{1,2, \ldots, n\})$. Then $\mathscr{T}$ can also be written as $\mathscr{T}=\mathscr{A}_{i}+\mathscr{M}_{i}+\mathscr{B}_{i}$ for each $i$. In this paper, if no confusion occurs, for any $A_{i} \in \mathscr{A}_{i}, M_{i} \in \mathscr{M}_{i}$ and $B_{i} \in \mathscr{B}_{i}$, we always identify

$$
\begin{gathered}
A_{i} \cong\left(\begin{array}{cccc}
r_{11} & m_{12} & \cdots & m_{1 i} \\
0 & r_{22} & \cdots & m_{2 i} \\
\vdots & \vdots & \ddots & \vdots \\
0 & 0 & \cdots & r_{i i}
\end{array}\right), \\
M_{i} \cong\left(\begin{array}{cccc}
m_{1, i+1} & m_{1, i+2} & \cdots & m_{1 n} \\
m_{2, i+1} & m_{2, i+2} & \cdots & m_{2 n} \\
\vdots & \vdots & \ddots & \vdots \\
m_{i, i+1} & m_{i, i+2} & \cdots & m_{i n}
\end{array}\right),
\end{gathered}
$$

and

$$
B_{i} \cong\left(\begin{array}{cccc}
r_{i+1, i+1} & m_{i+1, i+2} & \cdots & m_{i n} \\
0 & r_{i+2, i+2} & \cdots & m_{i+2, n} \\
\vdots & \vdots & \ddots & \vdots \\
0 & 0 & \cdots & r_{n n}
\end{array}\right) .
$$

\section{Proof of Theorem 1}

The "if" part is obvious. For the "only if" part, we will prove it by checking a series of claims. Firstly, let $i \in\{1,2, \ldots, n\}$.

Claim 1. For any $A_{i} \in \mathscr{A}_{i}, M_{i} \in \mathscr{M}_{i}$ and $B_{i} \in \mathscr{B}_{i}$, we have 
(i) $Q_{i} \delta\left(A_{i}\right) Q_{i}=0$;

(ii) $P_{i} \delta\left(B_{i}\right) P_{i}=0$;

(iii) $P_{i} \delta\left(M_{i}\right) P_{i}=Q_{i} \delta\left(M_{i}\right) Q_{i}=0$.

It is clear that $\delta(0)=0$. For any $A_{i} \in \mathscr{A}_{i}$, since $A_{i} \circ Q_{i}=0$, one gets

$$
\begin{aligned}
0 & =\delta\left(A_{i} \circ Q_{i}\right)=\delta\left(A_{i}\right) \circ Q_{i}+A_{i} \circ \delta\left(Q_{i}\right) \\
& =P_{i} \delta\left(A_{i}\right) Q_{i}+2 Q_{i} \delta\left(A_{i}\right) Q_{i}+A_{i} \delta\left(Q_{i}\right)+\delta\left(Q_{i}\right) A_{i} .
\end{aligned}
$$

Multiplying by $Q_{i}$ from the two sides in the above equation, and by the 2-torsion freeness of $\mathscr{T}$, one has $Q_{i} \delta\left(A_{i}\right) Q_{i}=0$.

Similarly, by using the relation $P_{i} \circ B_{i}=0$, one can show $P_{i} \delta\left(B_{i}\right) P_{i}=0$. So, the statements (i)-(ii) are true.

For any $M_{i} \in \mathscr{M}_{i}$, we have

$$
\begin{aligned}
& \delta\left(M_{i}\right)=\delta\left(P_{i} \circ M_{i}\right)=\delta\left(P_{i}\right) \circ M_{i}+P_{i} \circ \delta\left(M_{i}\right) \\
& \quad=\delta\left(P_{i}\right) M_{i}+M_{i} \delta\left(P_{i}\right)+2 P_{i} \delta\left(M_{i}\right) P_{i}+P_{i} \delta\left(M_{i}\right) Q_{i} .
\end{aligned}
$$

Multiplying by $P_{i}$ and $Q_{i}$ from the two sides in the above equation, respectively, one gets $P_{i} \delta\left(M_{i}\right) P_{i}=0$ and $Q_{i} \delta\left(M_{i}\right) Q_{i}=0$. So, the statement (iii) is true, and the claim holds.

Now, define two maps $d_{i}, \delta_{i}: \mathscr{T} \rightarrow \mathscr{T}$ by

$$
d_{i}(X)=\left[\delta\left(P_{i}\right), X\right] \text { and } \delta_{i}(X)=\delta(X)+d_{i}(X) .
$$

It is easy to check that $d_{i}$ is an additive derivation and $\delta_{i}$ is a multiplicative Jordan derivation. Then, Claim 1 is also true for $\delta_{i}$, that is,

$$
\begin{gathered}
Q_{i} \delta_{i}\left(A_{i}\right) Q_{i}=0, \quad P_{i} \delta_{i}\left(B_{i}\right) P_{i}=0, \\
P_{i} \delta_{i}\left(M_{i}\right) P_{i}=Q_{i} \delta_{i}\left(M_{i}\right) Q_{i}=0 .
\end{gathered}
$$

So

$\delta_{i}\left(P_{i}\right)=\delta\left(P_{i}\right)+d_{i}\left(P_{i}\right)=\delta\left(P_{i}\right)+\left[\delta\left(P_{i}\right), P_{i}\right]=P_{i} \delta\left(P_{i}\right) P_{i}$.

This yields that

$$
\delta_{i}\left(P_{i}\right)=P_{i} \delta\left(P_{i}\right) P_{i}=P_{i} \delta_{i}\left(P_{i}\right) P_{i} .
$$

We will discuss the properties of $\delta_{i}$ for $i \in\{1,2, \ldots, n\}$ by Claims $2-5$.

Claim 2. For any $A_{i} \in \mathscr{A}_{i}, M_{i} \in \mathscr{M}_{i}$, and $B_{i} \in \mathscr{B}_{i}$, we have

(i) $\delta_{i}\left(M_{i}\right) \in \mathscr{M}_{i}, \delta_{i}\left(A_{i}\right) \in \mathscr{A}_{i}$, and $\delta_{i}\left(B_{i}\right) \in \mathscr{B}_{i}$;

(ii) $\delta_{i}\left(P_{i}\right) M_{i}=M_{i} \delta_{i}\left(Q_{i}\right)=0$.
By (2), it is true that $\delta_{i}\left(M_{i}\right) \in \mathscr{M}_{i}$. By (2)-(3), one has

$0=\delta_{i}\left(P_{i} \circ Q_{i}\right)=\delta_{i}\left(P_{i}\right) \circ Q_{i}+P_{i} \circ \delta_{i}\left(Q_{i}\right)=P_{i} \delta_{i}\left(Q_{i}\right) Q_{i}$,

and so $\delta_{i}\left(Q_{i}\right)=Q_{i} \delta_{i}\left(Q_{i}\right) Q_{i}$. Thus, for any $A_{i} \in \mathscr{A}_{i}$, by (2) again, one obtains

$0=\delta_{i}\left(A_{i} \circ Q_{i}\right)=\delta_{i}\left(A_{i}\right) \circ Q_{i}+A_{i} \circ \delta_{i}\left(Q_{i}\right)=P_{i} \delta_{i}\left(A_{i}\right) Q_{i}$.

It follows that $\delta_{i}\left(A_{i}\right)=P_{i} \delta_{i}\left(A_{i}\right) P_{i} \in \mathscr{A}_{i}$.

Similarly, one can show $\delta_{i}\left(B_{i}\right)=Q_{i} \delta_{i}\left(B_{i}\right) Q_{i} \in$ $\mathscr{B}_{i}$ for all $B_{i} \in \mathscr{B}_{i}$. The statement (i) is true.

Finally, for any $M_{i} \in \mathscr{M}_{i}$, by Claim 2(i), one gets

$$
\begin{aligned}
\delta_{i}\left(M_{i}\right)=\delta_{i}\left(P_{i} \circ M_{i}\right) & =\delta_{i}\left(P_{i}\right) \circ M_{i}+P_{i} \circ \delta_{i}\left(M_{i}\right) \\
& =\delta_{i}\left(P_{i}\right) M_{i}+\delta_{i}\left(M_{i}\right)
\end{aligned}
$$

and

$$
\begin{aligned}
\delta_{i}\left(M_{i}\right)=\delta_{i}\left(M_{i} \circ Q_{i}\right) & =\delta_{i}\left(M_{i}\right) \circ Q_{i}+M_{i} \circ \delta_{i}\left(Q_{i}\right) \\
& =\delta_{i}\left(M_{i}\right)+M_{i} \delta_{i}\left(Q_{i}\right),
\end{aligned}
$$

which imply that $\delta_{i}\left(P_{i}\right) M_{i}=M_{i} \delta_{i}\left(Q_{i}\right)=0$. So, the statement (ii) is true.

Claim 3. For any $A_{i} \in \mathscr{A}_{i}, M_{i} \in \mathscr{M}_{i}$, and $B_{i} \in \mathscr{B}_{i}$, we have

(i) $\delta_{i}\left(A_{i}+M_{i}\right) \in \mathscr{A}_{i}+\mathscr{M}_{i}$ and $\delta_{i}\left(M_{i}\right)=P_{i} \delta_{i}\left(A_{i}+M_{i}\right) Q_{i}$;

(ii) $\delta_{i}\left(M_{i}+B_{i}\right) \in \mathscr{M}_{i}+\mathscr{B}_{i}$ and $\delta_{i}\left(M_{i}\right)=P_{i} \delta_{i}\left(M_{i}+B_{i}\right) Q_{i}$.

We only give the proof of (i). The proof of (ii) is similar and we omit it here. For any $A_{i} \in \mathscr{A}_{i}$ and $M_{i} \in \mathscr{M}_{i}$, by Claim 2, we get

$$
\begin{aligned}
\delta_{i}\left(M_{i}\right) & =\delta_{i}\left(M_{i} \circ Q_{i}\right)=\delta_{i}\left(\left(A_{i}+M_{i}\right) \circ Q_{i}\right) \\
& =\delta_{i}\left(A_{i}+M_{i}\right) \circ Q_{i}+A_{i} \circ \delta_{i}\left(Q_{i}\right)+M_{i} \circ \delta_{i}\left(Q_{i}\right) \\
& =P_{i} \delta_{i}\left(A_{i}+M_{i}\right) Q_{i}+2 Q_{i} \delta_{i}\left(A_{i}+M_{i}\right) Q_{i} .
\end{aligned}
$$

It follows from Claim 2(i) and 2-torsion freeness of $\mathscr{T}$ that $\delta_{i}\left(M_{i}\right)=P_{i} \delta_{i}\left(A_{i}+M_{i}\right) Q_{i}$ and $Q_{i} \delta_{i}\left(A_{i}+M_{i}\right) Q_{i}=0$.

Claim 4. For any $X=A_{i}+M_{i}+B_{i} \in \mathscr{T}$, we have

(i) $P_{i} \delta_{i}(X) Q_{i}=\delta_{i}\left(P_{i} X Q_{i}\right)$;

(ii) $E_{t}\left(\delta_{i}\left(A_{i}+M_{i}+B_{i}\right)-\delta_{i}\left(A_{i}\right)-\delta_{i}\left(M_{i}\right)-\delta_{i}\left(B_{i}\right)\right) E_{t}=$ 0 , where $t=1, \ldots, n$. 
For any $X=A_{i}+M_{i}+B_{i} \in \mathscr{T}$, Claim 2 gives

$\delta_{i}\left(2 A_{i}+M_{i}\right)=\delta_{i}\left(X \circ P_{i}\right)=\delta_{i}(X) \circ P_{i}+X \circ \delta_{i}\left(P_{i}\right)$

$=2 P_{i} \delta_{i}(X) P_{i}+P_{i} \delta_{i}(X) Q_{i}+A_{i} \delta_{i}\left(P_{i}\right) P_{i}+P_{i} \delta_{i}\left(P_{i}\right) A_{i}$

and

$$
\begin{aligned}
& \begin{array}{l}
\delta_{i}\left(2 A_{i}+M_{i}\right)= \\
=\delta_{i}\left(\left(A_{i}+M_{i}\right) \circ P_{i}\right) \\
=\delta_{i}\left(A_{i}+M_{i}\right) \circ P_{i}+\left(A_{i}+M_{i}\right) \circ \delta_{i}\left(P_{i}\right) \\
=2 P_{i} \delta_{i}\left(A_{i}+M_{i}\right) P_{i}+P_{i} \delta_{i}\left(A_{i}+M_{i}\right) Q_{i}+A_{i} \delta_{i}\left(P_{i}\right) P_{i}+P_{i} \delta_{i}\left(P_{i}\right) A_{i} .
\end{array}
\end{aligned}
$$

Comparing (4)-(5) and Claim 3(i) gives

$$
\begin{aligned}
P_{i} \delta_{i}(X) Q_{i} & =P_{i} \delta_{i}\left(A_{i}+M_{i}+B_{i}\right) Q_{i} \\
& =P_{i} \delta_{i}\left(A_{i}+M_{i}\right) Q_{i}=\delta_{i}\left(M_{i}\right)=\delta_{i}\left(P_{i} X Q_{i}\right)
\end{aligned}
$$

and

$$
2 P_{i} \delta_{i}(X) P_{i}=2 P_{i} \delta_{i}\left(A_{i}+M_{i}\right) P_{i} .
$$

Since $\mathscr{T}$ is 2-torsion free, (6) implies

$$
P_{i} \delta_{i}(X) P_{i}=P_{i} \delta_{i}\left(A_{i}+M_{i}\right) P_{i} .
$$

Next, take any $M_{i}^{\prime} \in \mathscr{M}_{i}$. Note that

$\delta_{i}\left(\left(A_{i}+M_{i}\right) \circ M_{i}^{\prime}\right)=\delta_{i}\left(A_{i}+M_{i}\right) \circ M_{i}^{\prime}+\left(A_{i}+M_{i}\right) \circ \delta_{i}\left(M_{i}^{\prime}\right)$

and

$\delta_{i}\left(\left(A_{i}+M_{i}\right) \circ M_{i}^{\prime}\right)=\delta_{i}\left(A_{i} \circ M_{i}^{\prime}\right)=\delta_{i}\left(A_{i}\right) \circ M_{i}^{\prime}+A_{i} \circ \delta_{i}\left(M_{i}^{\prime}\right)$.

Combining the above two equations, Claim 2(i) and Claim 3(i) yields

$\left(\delta_{i}\left(A_{i}+M_{i}\right)-\delta_{i}\left(A_{i}\right)-\delta_{i}\left(M_{i}\right)\right) M_{i}^{\prime}=0, \quad \forall M_{i}^{\prime} \in \mathscr{M}_{i}$.

Moreover, since $\mathscr{M}_{i j}$ is a faithful left $\mathscr{R}_{i}$-module, one can show that for $t=1, \ldots, i$,

$$
E_{t}\left(\delta_{i}\left(A_{i}+M_{i}\right)-\delta_{i}\left(A_{i}\right)-\delta_{i}\left(M_{i}\right)\right) E_{t}=0 .
$$

By the fact $P_{i} \delta_{i}\left(B_{i}\right) P_{i}=0$ and (7)-(8), one achieves, for $t=1,2, \ldots, i$,

$$
E_{t}\left(\delta_{i}\left(A_{i}+M_{i}+B_{i}\right)-\delta_{i}\left(A_{i}\right)-\delta_{i}\left(M_{i}\right)-\delta_{i}\left(B_{i}\right)\right) E_{t}=0 .
$$

Finally, by calculating $\delta_{i}\left(M_{i}^{\prime} \circ\left(M_{i}+B_{i}\right)\right)$, a similar argument to that of the above gives, for $t=i+1, \ldots, n$,

$$
E_{t}\left(\delta_{i}\left(A_{i}+M_{i}+B_{i}\right)-\delta_{i}\left(A_{i}\right)-\delta_{i}\left(M_{i}\right)-\delta_{i}\left(B_{i}\right)\right) E_{t}=0 .
$$

It follows from (9)-(10) that $E_{t}\left(\delta_{i}\left(A_{i}+M_{i}+B_{i}\right)-\right.$ $\left.\delta_{i}\left(A_{i}\right)-\delta_{i}\left(M_{i}\right)-\delta_{i}\left(B_{i}\right)\right) E_{t}=0$ holds for $t=1, \ldots, n$. The claim holds.
Claim 5. $\delta_{i}$ is additive on $\mathscr{M}_{i}$.

For $P_{i}$ and any $M_{i} \in \mathscr{M}_{i}$, on the one hand, by Claim 4(i), one has

$$
\begin{aligned}
& \delta_{i}\left(2 P_{i}\right)=\delta_{i}\left(\left(P_{i}+M_{i}\right) \circ\left(P_{i}-Q_{i}\right)\right) \\
& \quad=\delta_{i}\left(P_{i}+M_{i}\right) \circ\left(P_{i}-Q_{i}\right)+\left(P_{i}+M_{i}\right) \circ \delta_{i}\left(P_{i}-Q_{i}\right) \\
& \quad=2 P_{i} \delta_{i}\left(P_{i}+M_{i}\right) P_{i}+2 P_{i} \delta_{i}\left(P_{i}-Q_{i}\right) P_{i} \\
& +P_{i} \delta_{i}\left(P_{i}-Q_{i}\right) Q_{i}+M_{i} \delta_{i}\left(P_{i}-Q_{i}\right) Q_{i}+P_{i} \delta_{i}\left(P_{i}-Q_{i}\right) M_{i} .
\end{aligned}
$$

On the other hand, by Claim 2(i) and Claim 4(i), one has

$$
\begin{aligned}
& \delta_{i}\left(2 P_{i}\right)=\delta_{i}\left(P_{i} \circ\left(P_{i}-Q_{i}\right)\right) \\
& \quad=\delta_{i}\left(P_{i}\right) \circ\left(P_{i}-Q_{i}\right)+P_{i} \circ \delta_{i}\left(P_{i}-Q_{i}\right) \\
& \quad=2 P_{i} \delta_{i}\left(P_{i}\right) P_{i}+2 P_{i} \delta_{i}\left(P_{i}-Q_{i}\right) P_{i}+P_{i} \delta_{i}\left(P_{i}-Q_{i}\right) Q_{i} \\
& \quad=2 \delta_{i}\left(P_{i}\right)+2 P_{i} \delta_{i}\left(P_{i}-Q_{i}\right) P_{i} .
\end{aligned}
$$

Comparing the above two equations yields

$$
P_{i} \delta_{i}\left(P_{i}+M_{i}\right) P_{i}=\delta_{i}\left(P_{i}\right)
$$

by the 2-torsion freeness of $\mathscr{T}$, which together with Claim 3(i) and Claim 4(i), implies that

$$
\begin{aligned}
\delta_{i}\left(P_{i}+M_{i}\right) & =P_{i} \delta_{i}\left(P_{i}+M_{i}\right) P_{i}+P_{i} \delta_{i}\left(P_{i}+M_{i}\right) Q_{i} \\
& =\delta_{i}\left(P_{i}\right)+\delta_{i}\left(M_{i}\right) .
\end{aligned}
$$

Symmetrically, one can show that

$$
\delta_{i}\left(M_{i}+Q_{i}\right)=\delta_{i}\left(M_{i}\right)+\delta_{i}\left(Q_{i}\right) .
$$

Now, for any $M_{i}, M_{i}^{\prime} \in \mathscr{M}_{i}$, by Claim 2 and (11)-(12), one achieves

$$
\begin{aligned}
\delta_{i}\left(M_{i}\right. & \left.+M_{i}^{\prime}\right)=\delta_{i}\left(\left(P_{i}+M_{i}\right) \circ\left(M_{i}^{\prime}+Q_{i}\right)\right) \\
= & \delta_{i}\left(P_{i}+M_{i}\right) \circ\left(M_{i}^{\prime}+Q_{i}\right)+\left(P_{i}+M_{i}\right) \circ \delta_{i}\left(M_{i}^{\prime}+Q_{i}\right) \\
= & \left(\delta_{i}\left(P_{i}\right)+\delta_{i}\left(M_{i}\right)\right) \circ\left(M_{i}^{\prime}+Q_{i}\right) \\
& +\left(P_{i}+M_{i}\right) \circ\left(\delta_{i}\left(M_{i}^{\prime}\right)+\delta_{i}\left(Q_{i}\right)\right) \\
= & \delta_{i}\left(M_{i}\right)+\delta_{i}\left(M_{i}^{\prime}\right),
\end{aligned}
$$

that is, $\delta_{i}$ is additive on $\mathscr{M}_{i}$, completing the proof of the claim.

From now on, let $i \in\{2, \ldots, n-1\}$. Define another maps $\tau_{i}: \mathscr{T} \rightarrow \mathscr{T}$ by

$$
\tau_{i}(X)=\delta_{1}(X)+\left[\delta_{1}\left(P_{i}\right), X\right] .
$$

By the same arguments as those of Claims 2-5 for $\delta_{i}$, we can prove that $\tau_{i}$ is also a multiplicative Jordan derivation satisfying $\tau_{i}\left(P_{i}\right)=P_{i} \tau_{i}\left(P_{i}\right) P_{i}$, 
and Claims 2-5 still hold for the map $\tau_{i}$.

Claim 6. For any $M_{i} \in \mathscr{M}_{i}$, we have $\delta_{1}\left(M_{i}\right)=\tau_{i}\left(M_{i}\right)$. Therefore, $\delta_{1}$ is additive on $\mathscr{M}_{i}$.

Take any $M_{i} \in \mathscr{M}_{i} . \quad$ As $\delta_{1}\left(M_{i}\right)=\tau_{i}\left(M_{i}\right)-$ $\left[\delta_{1}\left(P_{i}\right), M_{i}\right] \in \mathscr{M}_{i}$, it is true that

$$
\begin{aligned}
P_{i} \delta_{1}\left(M_{i}\right) Q_{i} & =\delta_{1}\left(M_{i}\right)=\delta_{1}\left(P_{i} \circ M_{i}\right) \\
& =\delta_{1}\left(P_{i}\right) \circ M_{i}+P_{i} \circ \delta_{1}\left(M_{i}\right) \\
& =\delta_{1}\left(P_{i}\right) M_{i}+M_{i} \delta_{1}\left(P_{i}\right)+P_{i} \delta_{1}\left(M_{i}\right) Q_{i} .
\end{aligned}
$$

This means

$$
\delta_{1}\left(P_{i}\right) M_{i}+M_{i} \delta_{1}\left(P_{i}\right)=0 .
$$

Also note that

$$
\begin{aligned}
\mathscr{A}_{i} \ni \tau_{i}\left(P_{i}\right) & =\delta_{1}\left(P_{i}\right)+\left[\delta_{1}\left(P_{i}\right), P_{i}\right] \\
& =P_{i} \delta_{1}\left(P_{i}\right) P_{i}+Q_{i} \delta_{1}\left(P_{i}\right) Q_{i},
\end{aligned}
$$

which implies $Q_{i} \delta_{1}\left(P_{i}\right) Q_{i}=0$. So

$$
M_{i} \delta_{1}\left(P_{i}\right)=0=\delta_{1}\left(P_{i}\right) M_{i}, \quad \forall M_{i} \in \mathscr{M}_{i} .
$$

It follows that $\delta_{1}\left(M_{i}\right)=\tau_{i}\left(M_{i}\right)$. Hence $\delta_{1}$ is additive on $\mathscr{M}_{i}$.

Claim 7. For any $X=A_{1}+M_{1}+B_{1} \in \mathscr{T}$, we have $\delta_{1}(X)=\delta_{1}\left(A_{1}\right)+\delta_{1}\left(M_{1}\right)+\delta_{1}\left(B_{1}\right)$.

For any $X=A_{1}+M_{1}+B_{1}=A_{i}+M_{i}+B_{i} \in \mathscr{T}$, let

$$
\begin{aligned}
H_{1} & =\delta_{1}(X)-\delta_{1}\left(A_{1}\right)-\delta_{1}\left(M_{1}\right)-\delta_{1}\left(B_{1}\right) \quad \text { and } \\
K_{i} & =\tau_{i}(X)-\tau_{i}\left(A_{i}\right)-\tau_{i}\left(M_{i}\right)-\tau_{i}\left(B_{i}\right) .
\end{aligned}
$$

Our goal is to show that $H_{1}=0$. In fact, by (13), one has

$$
\begin{aligned}
H_{1}- & K_{i}=\delta_{1}(X)-\tau_{i}(X)-\delta_{1}\left(A_{1}\right)+\tau_{i}\left(A_{i}\right) \\
& -\delta_{1}\left(M_{1}\right)+\tau_{i}\left(M_{i}\right)-\delta_{1}\left(B_{1}\right)+\tau_{i}\left(B_{i}\right) \\
= & -\left[\delta_{1}\left(P_{i}\right), X\right]-\left(\tau_{i}\left(A_{1}\right)-\left[\delta_{1}\left(P_{i}\right), A_{1}\right]\right)+\tau_{i}\left(A_{i}\right) \\
& -\left(\tau_{i}\left(M_{1}\right)-\left[\delta_{1}\left(P_{i}\right), M_{1}\right]\right)+\left(\delta_{1}\left(M_{i}\right)+\left[\delta_{1}\left(P_{i}\right), M_{i}\right]\right) \\
& -\left(\tau_{i}\left(B_{1}\right)-\left[\delta_{1}\left(P_{i}\right), B_{1}\right]\right)+\tau_{i}\left(B_{i}\right) \\
= & -\tau_{i}\left(A_{1}\right)+\tau_{i}\left(A_{i}\right)-\tau_{i}\left(M_{1}\right)+\delta_{1}\left(M_{i}\right) \\
& +\left[\delta_{1}\left(P_{i}\right), M_{i}\right]-\tau_{i}\left(B_{1}\right)+\tau_{i}\left(B_{i}\right) .
\end{aligned}
$$

Observe that, by Claim 2(i) for $\tau_{i}$, one gets

$$
\begin{gathered}
\tau_{i}\left(A_{1}\right)=P_{i} \tau_{i}\left(A_{1}\right) P_{i}, \quad \tau_{i}\left(A_{i}\right)=P_{i} \tau_{i}\left(A_{i}\right) P_{i}, \\
\tau_{i}\left(B_{i}\right)=Q_{i} \tau_{i}\left(B_{i}\right) Q_{i}
\end{gathered}
$$

by (13) and Claim 2(i) for $\delta_{1}$, one has

$$
\tau_{i}\left(M_{1}\right)=\delta_{1}\left(M_{1}\right)+\left[\delta_{1}\left(P_{i}\right), M_{1}\right] \in \mathscr{M}_{1} ;
$$

and by Claim 6 and Claim 2(i) for $\delta_{1}$, one gets

$$
\begin{aligned}
\delta_{1}\left(M_{i}\right) & =\delta_{1}\left(P_{1} M_{i} P_{1}\right)+\delta_{1}\left(P_{1} M_{i} Q_{1}\right)+\delta_{1}\left(Q_{1} M_{i} Q_{1}\right) \\
& =\delta_{1}\left(P_{1} M_{i} Q_{1}\right)+\delta_{1}\left(Q_{1} M_{i} Q_{1}\right) \\
& \in \mathscr{M}_{1}+\delta_{1}\left(Q_{1} M_{i} Q_{1}\right) .
\end{aligned}
$$

Now, combining Claim 4(i) and (13)-(18) yields

$$
\begin{aligned}
E_{i}\left(H_{1}-K_{i}\right) E_{j} & =E_{i} \delta_{1}\left(M_{i}\right) E_{j}-E_{i} \tau_{i}\left(B_{1}\right) E_{j} \\
& =E_{i} \delta_{1}\left(Q_{1} M_{i} Q_{1}\right) E_{j}-E_{i} P_{i} \tau_{i}\left(B_{1}\right) Q_{i} E_{j} \\
& =E_{i} \delta_{1}\left(Q_{1} M_{i} Q_{1}\right) E_{j}-E_{i} \tau_{i}\left(P_{i} B_{1} Q_{i}\right) E_{j} \\
& =E_{i}\left(\delta_{1}\left(Q_{1} M_{i} Q_{1}\right)-\tau_{i}\left(Q_{1} M_{i} Q_{1}\right)\right) E_{j} \\
& =E_{i}\left[Q_{1} M_{i} Q_{1}, \delta_{1}\left(P_{i}\right)\right] E_{j}=0
\end{aligned}
$$

where $j=i+1, \ldots, n$. Here, the reciprocal 3rd equation is due to $Q_{1} M_{i} Q_{1}=P_{i} B_{1} Q_{i} \in \sum_{k=2}^{i} \sum_{l=1}^{n-i} \mathscr{T}_{k, i+l}$.

On the other hand, by Claim 4(i) and Claim 2(i) for $\tau_{i}$, we know that $P_{i} K_{i} Q_{i}=0$, which implies $E_{i} K_{i} E_{j}=E_{i} P_{i} K_{i} Q_{i} E_{j}=0$ for $j=i+1, \ldots, n$. Hence (19) reduces to $E_{i} H_{1} E_{j}=0$.

Also note that $P_{1} H_{1} Q_{1}=0$ and $E_{t} H_{1} E_{t}=0$, $t=1, \ldots, n$, by Claim 4 and Claim 2(i) for $\delta_{1}$. Hence $H_{1}=0$, completing the proof of the claim.

Claim 8. $\delta_{1}$ is additive on $\mathscr{T}$.

We will prove the claim by several steps.

Step 8.1. $\delta_{1}$ is additive on $\mathscr{M}_{1}$. By Claim 5, this is true.

Step 8.2. $\delta_{1}$ is additive on $\mathscr{A}_{1}$.

Take any $A_{1}, A_{1}^{\prime} \in \mathscr{A}_{1}$ and any $M_{1} \in \mathscr{M}_{1}$. By Claim 2(i) and Step 8.1, we have

$$
\begin{aligned}
\delta_{1}\left(\left(A_{1}\right.\right. & \left.\left.+A_{1}^{\prime}\right) \circ M_{1}\right) \\
& =\delta_{1}\left(A_{1}+A_{1}^{\prime}\right) \circ M_{1}+\left(A_{1}+A_{1}^{\prime}\right) \circ \delta_{1}\left(M_{1}\right) \\
& =\delta_{1}\left(A_{1}+A_{1}^{\prime}\right) M_{1}+\left(A_{1}+A_{1}^{\prime}\right) \delta_{1}\left(M_{1}\right)
\end{aligned}
$$

and

$$
\begin{aligned}
& \delta_{1}\left(\left(A_{1}+A_{1}^{\prime}\right) \circ M_{1}\right)=\delta_{1}\left(A_{1} M_{1}+A_{1}^{\prime} M_{1}\right) \\
& \quad=\delta_{1}\left(A_{1} \circ M_{1}\right)+\delta_{1}\left(A_{1}^{\prime} \circ M_{1}\right) \\
& \quad=\delta_{1}\left(A_{1}\right) M_{1}+A_{1} \delta_{1}\left(M_{1}\right)+\delta_{1}\left(A_{1}^{\prime}\right) M_{1}+A_{1}^{\prime} \delta_{1}\left(M_{1}\right) .
\end{aligned}
$$

Combining the above two equations gives $\left(\delta_{1}\left(A_{1}+A_{1}^{\prime}\right)-\delta_{1}\left(A_{1}\right)-\delta_{1}\left(A_{1}^{\prime}\right)\right) M_{1}=0 \quad$ for all 
$M_{1} \in \mathscr{M}_{1}$. Since $\mathscr{M}_{1 j}$ is a faithful left $\mathscr{R}_{1}$-module, we get $\delta_{1}\left(A_{1}+A_{1}^{\prime}\right)-\delta_{1}\left(A_{1}\right)-\delta_{1}\left(A_{1}^{\prime}\right)=0$.

Step 8.3. $\delta_{1}$ is additive on $\mathscr{B}_{1}$.

For any $B_{1}, B_{1}^{\prime} \in \mathscr{B}_{1}$ and any $M_{1} \in \mathscr{M}_{1}$, by Claim 8.1, one obtains

$$
\begin{aligned}
\delta_{1}\left(M_{1} \circ\right. & \left.\left(B_{1}+B_{1}^{\prime}\right)\right) \\
& =\delta_{1}\left(M_{1}\right) \circ\left(B_{1}+B_{1}^{\prime}\right)+M_{1} \circ \delta_{1}\left(B_{1}+B_{1}^{\prime}\right) \\
& =\delta_{1}\left(M_{1}\right)\left(B_{1}+B_{1}^{\prime}\right)+M_{1} \delta_{1}\left(B_{1}+B_{1}^{\prime}\right)
\end{aligned}
$$

and

$$
\begin{aligned}
& \delta_{1}\left(M_{1} \circ\left(B_{1}+B_{1}^{\prime}\right)\right)=\delta_{1}\left(M_{1} B_{1}+M_{1} B_{1}^{\prime}\right) \\
& \quad=\delta_{1}\left(M_{1} \circ B_{1}\right)+\delta_{1}\left(M_{1} \circ B_{1}^{\prime}\right) \\
& \quad=\delta_{1}\left(M_{1}\right) B_{1}+M_{1} \delta_{1}\left(B_{1}\right)+\delta_{1}\left(M_{1}\right) B_{1}^{\prime}+M_{1} \delta_{1}\left(B_{1}\right),
\end{aligned}
$$

which implies $M_{1}\left(\delta_{1}\left(B_{1}+B_{1}^{\prime}\right)-\delta_{1}\left(B_{1}\right)-\delta_{1}\left(B_{1}^{\prime}\right)\right)=0$. It follows from the fact $\mathscr{M}_{1 t}$ is a faithful right $\mathscr{R}_{t^{-}}$ module that, for $t=2, \ldots, n$,

$$
E_{t}\left(\delta_{1}\left(B_{1}+B_{1}^{\prime}\right)-\delta_{1}\left(B_{1}\right)-\delta_{1}\left(B_{1}^{\prime}\right)\right) E_{t}=0 .
$$

On the other hand, by Claim 4(i) for $\tau_{i}$, Claim 5 for $\tau_{i}$, and (13), one obtains

$$
\begin{aligned}
P_{i} \delta_{1}\left(B_{1}+\right. & \left.B_{1}^{\prime}\right) Q_{i}=P_{i}\left(\tau_{i}\left(B_{1}+B_{1}^{\prime}\right)-\left[\delta_{1}\left(P_{i}\right), B_{1}+B_{1}^{\prime}\right]\right) Q_{i} \\
= & P_{i} \tau_{i}\left(B_{1}+B_{1}^{\prime}\right) Q_{i}-P_{i}\left(\left[\delta_{1}\left(P_{i}\right), B_{1}+B_{1}^{\prime}\right]\right) Q_{i} \\
= & \tau_{i}\left(P_{i}\left(B_{1}+B_{1}^{\prime}\right) Q_{i}\right)-P_{i}\left(\left[\delta_{1}\left(P_{i}\right), B_{1}\right]\right) Q_{i} \\
& -P_{i}\left(\left[\delta_{1}\left(P_{i}\right), B_{1}^{\prime}\right]\right) Q_{i} \\
= & \tau_{i}\left(P_{i} B_{1} Q_{i}\right)-P_{i}\left(\left[\delta_{1}\left(P_{i}\right), B_{1}\right]\right) Q_{i}+\tau_{i}\left(P_{i} B_{1}^{\prime} Q_{i}\right) \\
& -P_{i}\left(\left[\delta_{1}\left(P_{i}\right), B_{1}^{\prime}\right]\right) Q_{i} \\
= & P_{i}\left(\tau_{i}\left(B_{1}\right)-\left[\delta_{1}\left(P_{i}\right), B_{1}\right]\right) Q_{i} \\
& +P_{i}\left(\tau_{i}\left(B_{1}^{\prime}\right)-\left[\delta_{1}\left(P_{i}\right), B_{1}^{\prime}\right]\right) Q_{i} \\
= & P_{i} \delta_{1}\left(B_{1}\right) Q_{i}+P_{i} \delta_{1}\left(B_{1}^{\prime}\right) Q_{i},
\end{aligned}
$$

which implies that, for $i=2, \ldots, n-1$,

$$
P_{i}\left(\delta_{1}\left(B_{1}+B_{1}^{\prime}\right)-\delta_{1}\left(B_{1}\right)-\delta_{1}\left(B_{1}^{\prime}\right)\right) Q_{i}=0 .
$$

Combining (20)-(21) yields $\delta_{1}\left(B_{1}+B_{1}^{\prime}\right)-\delta_{1}\left(B_{1}\right)-$ $\delta_{1}\left(B_{1}^{\prime}\right)=0$.

Step 8.4. $\delta_{1}$ is additive on $\mathscr{T}$.

For any $X_{1}=A_{1}+M_{1}+B_{1} \in \mathscr{T}$ and $X_{2}=A_{1}^{\prime}+M_{1}^{\prime}+$ $B_{1}^{\prime} \in \mathscr{T}$, by Claim 7 and Steps 8.1-8.3, we achieve

$$
\begin{aligned}
\delta_{1}\left(X_{1}+\right. & \left.X_{2}\right)=\delta_{1}\left(A_{1}+M_{1}+B_{1}+A_{1}^{\prime}+M_{1}^{\prime}+B_{1}^{\prime}\right) \\
= & \delta_{1}\left(A_{1}+A_{1}^{\prime}\right)+\delta_{1}\left(M_{1}+M_{1}^{\prime}\right)+\delta_{1}\left(B_{1}+B_{1}^{\prime}\right) \\
= & \delta_{1}\left(A_{1}\right)+\delta_{1}\left(M_{1}\right)+\delta_{1}\left(B_{1}\right)+\delta_{1}\left(A_{1}^{\prime}\right) \\
& +\delta_{1}\left(M_{1}^{\prime}\right)+\delta_{1}\left(B_{1}^{\prime}\right) \\
= & \delta_{1}\left(X_{1}\right)+\delta_{1}\left(X_{2}\right) .
\end{aligned}
$$

Claim 9. $\delta_{1}$ is a derivation on $\mathscr{T}$.

We will prove the claim by several steps.

Step 9.1. For any $M_{1}, M_{1}^{\prime} \in \mathscr{M}_{1}$, we have $\delta_{1}\left(M_{1} M_{1}^{\prime}\right)=\delta_{1}\left(M_{1}\right) M_{1}^{\prime}+M_{1} \delta_{1}\left(M_{1}^{\prime}\right)=0$.

This is obvious by the fact $\delta_{1}\left(\mathscr{M}_{1}\right) \subseteq \mathscr{M}_{1}$.

Step 9.2. For any $A_{1}, A_{1}^{\prime} \in \mathscr{A}_{1}$ and any $M_{1} \in \mathscr{M}_{1}$, we have

$$
\begin{aligned}
\delta_{1}\left(A_{1} M_{1}\right) & =\delta_{1}\left(A_{1}\right) M_{1}+A_{1} \delta_{1}\left(M_{1}\right) \quad \text { and } \\
\delta_{1}\left(A_{1} A_{1}^{\prime}\right) & =\delta_{1}\left(A_{1}\right) A_{1}^{\prime}+A_{1} \delta_{1}\left(A_{1}^{\prime}\right) .
\end{aligned}
$$

Take any $A_{1}, A_{1}^{\prime} \in \mathscr{A}_{1}$ and any $M_{1} \in \mathscr{M}_{1}$. By Claim 2(i), one has

$$
\begin{aligned}
\delta_{1}\left(A_{1} A_{1}^{\prime} M_{1}\right) & =\delta_{1}\left(\left(A_{1} A_{1}^{\prime}\right) \circ M_{1}\right) \\
& =\delta_{1}\left(A_{1} A_{1}^{\prime}\right) \circ M_{1}+\left(A_{1} A_{1}^{\prime}\right) \circ \delta_{1}\left(M_{1}\right) \\
& =\delta_{1}\left(A_{1} A_{1}^{\prime}\right) M_{1}+A_{1} A_{1}^{\prime} \delta_{1}\left(M_{1}\right) ;
\end{aligned}
$$

particularly, we have $\delta_{1}\left(A_{1} M_{1}\right)=\delta_{1}\left(A_{1}\right) M_{1}+$ $A_{1} \delta_{1}\left(M_{1}\right)$. On the other hand, one gets

$$
\begin{gathered}
\delta_{1}\left(A_{1} A_{1}^{\prime} M_{1}\right)=\delta_{1}\left(A_{1} \circ A_{1}^{\prime} M_{1}\right) \\
=\delta_{1}\left(A_{1}\right) \circ\left(A_{1}^{\prime} M_{1}\right)+A_{1} \circ \delta_{1}\left(A_{1}^{\prime} M_{1}\right) \\
=\delta_{1}\left(A_{1}\right) A_{1}^{\prime} M_{1}+A_{1}\left(\delta_{1}\left(A_{1}^{\prime}\right) M_{1}+A_{1}^{\prime} \delta_{1}\left(M_{1}\right)\right) \\
=\delta_{1}\left(A_{1}\right) A_{1}^{\prime} M_{1}+A_{1} \delta_{1}\left(A_{1}^{\prime}\right) M_{1}+A_{1} A_{1}^{\prime} \delta_{1}\left(M_{1}\right) .
\end{gathered}
$$

combining (22)-(23) yields $\left(\delta_{1}\left(A_{1} A_{1}^{\prime}\right)-\delta_{1}\left(A_{1}\right) A_{1}^{\prime}-\right.$ $\left.A_{1} \delta_{1}\left(A_{1}^{\prime}\right)\right) M_{1}=0$ for all $M_{1} \in \mathscr{M}_{1}$. Since $\mathscr{M}_{1 j}$ is a faithful left $\mathscr{R}_{1}$-module, we obtain $\delta_{1}\left(A_{1} A_{1}^{\prime}\right)=\delta_{1}\left(A_{1}\right) A_{1}^{\prime}+A_{1} \delta_{1}\left(A_{1}^{\prime}\right)$.

Step 9.3. For any $B_{1}, B_{1}^{\prime} \in \mathscr{B}_{1}$ and any $M_{1} \in \mathscr{M}_{1}$, we have

$$
\begin{aligned}
\delta_{1}\left(M_{1} B_{1}\right) & =\delta_{1}\left(M_{1}\right) B_{1}+M_{1} \delta_{1}\left(B_{1}\right) \quad \text { and } \\
\delta_{1}\left(B_{1} B_{1}^{\prime}\right) & =\delta_{1}\left(B_{1}\right) B_{1}^{\prime}+B_{1} \delta_{1}\left(B_{1}^{\prime}\right) .
\end{aligned}
$$

Taking any $B_{1}, B_{1}^{\prime} \in \mathscr{B}_{1}$ and any $M_{1} \in \mathscr{M}_{1}$, by the same arguments as those of Step 9.2, we can prove that

$$
\begin{aligned}
& \delta_{1}\left(M_{1} B_{1}\right)=\delta_{1}\left(M_{1}\right) B_{1}+M_{1} \delta_{1}\left(B_{1}\right) \text { and } \\
& M_{1}\left(\delta_{1}\left(B_{1} B_{1}^{\prime}\right)-\delta_{1}\left(B_{1}\right) B_{1}^{\prime}-B_{1} \delta_{1}\left(B_{1}^{\prime}\right)\right)=0 .
\end{aligned}
$$

Since $\mathscr{M}_{i j}$ is a faithful right $\mathscr{R}_{j}$-module, one gets for $t=2, \ldots, n$,

$$
E_{t}\left(\delta_{1}\left(B_{1} B_{1}^{\prime}\right)-\delta_{1}\left(B_{1}\right) B_{1}^{\prime}-B_{1} \delta_{1}\left(B_{1}^{\prime}\right)\right) E_{t}=0 .
$$

Now, writing $B_{1}=\left(b_{k l}\right)_{n \times n}$ and $B_{1}^{\prime}=\left(b_{s t}^{\prime}\right)_{n \times n}$; then

$$
B_{1} B_{1}^{\prime}=\sum_{\substack{2 \leqslant k \leqslant l \leqslant n, 2 \leqslant s \leqslant t \leqslant n}} B_{k l} B_{s t}^{\prime}=\sum_{2 \leqslant k \leqslant l \leqslant t \leqslant n} B_{k l} B_{l t}^{\prime},
$$


where $B_{k l}$ is the element with $(k, l)$ position $b_{k, l}$ and other positions 0 . Thus, to show that $\delta_{1}\left(B_{1} B_{1}^{\prime}\right)=$ $\delta_{1}\left(B_{1}\right) B_{1}^{\prime}+B_{1} \delta_{1}\left(B_{1}^{\prime}\right)$, by the additivity of $\delta_{1}$, one only needs to check that $\delta_{1}$ satisfies the following equations:

$$
\left\{\begin{array}{l}
\delta_{1}\left(B_{k l} B_{l t}^{\prime}\right)=\delta_{1}\left(B_{k l}\right) B_{l t}^{\prime}+B_{k l} \delta_{1}\left(B_{l t}^{\prime}\right) \text { and } \\
\delta_{1}\left(B_{k l}\right) B_{s t}^{\prime}+B_{k l} \delta_{1}\left(B_{s t}^{\prime}\right)=0,
\end{array}\right.
$$

for all $2 \leqslant k \leqslant l \leqslant t \leqslant n$ and $2 \leqslant k \leqslant l \leqslant n, 2 \leqslant s \leqslant$ $t \leqslant n$ with $l \neq s$, respectively.

Observe that, by taking $B_{1}=B_{k k}$ and $B_{1}^{\prime}=E_{k}$ with $k \neq t$ in (24), one achieves

$$
\begin{aligned}
0 & =E_{t}\left(\delta_{1}\left(B_{k k} E_{k}\right)-\delta_{1}\left(B_{k k}\right) E_{k}-B_{k k} \delta_{1}\left(E_{k}\right)\right) E_{t} \\
& =E_{t} \delta_{1}\left(B_{k k}\right) E_{t},
\end{aligned}
$$

that is,

$$
E_{t} \delta_{1}\left(B_{k k}\right) E_{t}=0, \quad t, k \in\{2, \ldots, n\}, t \neq k .
$$

Step 9.3.1. For any $B_{k k}, B_{s s}^{\prime}$ with $k<s$, we have

$$
\begin{gathered}
0=\delta_{1}\left(B_{k k}\right) B_{s s}^{\prime}+B_{k k} \delta_{1}\left(B_{s s}^{\prime}\right) \quad \text { and } \\
\delta_{1}\left(B_{s s}^{\prime}\right) B_{k k}=B_{s s}^{\prime} \delta_{1}\left(B_{k k}\right)=0 .
\end{gathered}
$$

For $B_{k k}$ and $B_{s s}^{\prime}$ with $k<s$, we have

$$
\begin{aligned}
0=\delta_{1}\left(B_{k k} \circ B_{s s}^{\prime}\right)= & \delta_{1}\left(B_{k k}\right) \circ B_{s s}^{\prime}+B_{k k} \circ \delta_{1}\left(B_{s s}^{\prime}\right) \\
= & \delta_{1}\left(B_{k k}\right) B_{s s}^{\prime}+B_{s s}^{\prime} \delta_{1}\left(B_{k k}\right) \\
& +B_{k k} \delta_{1}\left(B_{s s}^{\prime}\right)+\delta_{1}\left(B_{s s}^{\prime}\right) B_{k k} .
\end{aligned}
$$

Note that (26) implies

$$
\begin{aligned}
& \delta_{1}\left(B_{k k}\right) B_{s s}^{\prime} \in \sum_{j=2}^{s-1} \mathscr{T}_{j s}, B_{s s}^{\prime} \delta_{1}\left(B_{k k}\right) \\
& \in \sum_{j=s+1}^{n} \mathscr{T}_{s j}, B_{k k} \delta_{1}\left(B_{s s}^{\prime}\right) \\
& \quad \in \sum_{j=k+1}^{n} \mathscr{T}_{k j}, \delta_{1}\left(B_{s s}^{\prime}\right) B_{k k} \in \sum_{j=2}^{k-1} \mathscr{T}_{j k} .
\end{aligned}
$$

These and (27) mean that $\delta_{1}\left(B_{k k}\right) B_{s s}^{\prime}+B_{k k} \delta_{1}\left(B_{s s}^{\prime}\right)=$ $0=B_{s s}^{\prime} \delta_{1}\left(B_{k k}\right)=\delta_{1}\left(B_{s s}^{\prime}\right) B_{k k}$.

Note that, by Step 9.3.1, one can check that, for $k=2, \ldots, n$,

$$
\delta_{1}\left(B_{k k}\right) \in \mathscr{T}_{2 k}+\cdots+\mathscr{T}_{k k}+\mathscr{T}_{k(k+1)}+\cdots+\mathscr{T}_{k n} .
$$

Step 9.3.2. For any $B_{k l}, B_{s t}^{\prime}$ with $k \leqslant l$ and $s \leqslant t$, we have

(i) $B_{s t}^{\prime} \delta_{1}\left(B_{k l}\right)=\delta_{1}\left(B_{s t}^{\prime}\right) B_{k l}=0$ if $k<s$ or $k=s$,
$s<t$ (ii) $\delta_{1}\left(B_{k l}\right) B_{s t}^{\prime}=B_{k l} \delta_{1}\left(B_{s t}^{\prime}\right)=0$ if $k>s$ or $k=s$, $k<l$.

Note that, by Claim 6 (that is, $\delta_{1}\left(M_{i}\right)=\tau_{i}\left(M_{i}\right)$ for all $M_{i}$ ), we know that $\delta_{1}\left(B_{k l}\right) \subseteq \mathscr{M}_{k} \cap \mathscr{B}_{1}$ holds for all $B_{k l}$ with $k<l$. In addition, by (28), it is true that $B_{s t}^{\prime} \delta_{1}\left(B_{k k}\right)=0$ for $k<s$. Now, the step is easily checked.

Step 9.3.3. For any $B_{k k}$ and $B_{k k}^{\prime}$, we have $\delta_{1}\left(B_{k k} B_{k k}^{\prime}\right)=\delta_{1}\left(B_{k k}\right) B_{k k}^{\prime}+B_{k k} \delta_{1}\left(B_{k k}^{\prime}\right)$.

For any $B_{k k}, B_{k k}^{\prime}$, by Claim 8 , one gets

$$
\begin{aligned}
2 \delta_{1}\left(B_{k k}\right)= & \delta_{1}\left(B_{k k} \circ E_{k}\right) \\
= & \delta_{1}\left(B_{k k}\right) \circ E_{k}+B_{k k} \circ \delta_{1}\left(E_{k}\right) \\
= & \delta_{1}\left(B_{k k}\right) E_{k}+E_{k} \delta_{1}\left(B_{k k}\right)+B_{k k} \delta_{1}\left(E_{k}\right) \\
& +\delta_{1}\left(E_{k}\right) B_{k k} .
\end{aligned}
$$

Multiplying respectively by $E_{t}, t=2, \ldots, k-1$, and $E_{k}$ from the left and the right in (29), one obtains

$$
E_{t} \delta_{1}\left(B_{k k}\right) E_{k}=E_{t} \delta_{1}\left(E_{k}\right) B_{k k}, t=2, \ldots, k-1 ;(30)
$$

multiplying respectively by $E_{k}$ and $E_{s}, s=k+$ $1, \ldots, n$, from the left and the right in (29), we get

$$
E_{k} \delta_{1}\left(B_{k k}\right) E_{s}=B_{k k} \delta_{1}\left(E_{k}\right) E_{s}, s=k+1, \ldots, n .
$$

Thus, (30)-(31) imply

$$
\begin{aligned}
& E_{t}\left(\delta_{1}\left(B_{k k} B_{k k}^{\prime}\right)-\delta_{1}\left(B_{k k}\right) B_{k k}^{\prime}-B_{k k} \delta_{1}\left(B_{k k}^{\prime}\right)\right) E_{k} \\
& \quad=E_{t} \delta_{1}\left(B_{k k} B_{k k}^{\prime}\right) E_{k}-E_{t} \delta_{1}\left(B_{k k}\right) B_{k k}^{\prime} \\
& \quad=E_{t} \delta_{1}\left(E_{k}\right) B_{k k} B_{k k}^{\prime}-E_{t} \delta_{1}\left(E_{k}\right) B_{k k} B_{k k}^{\prime}=0
\end{aligned}
$$

and

$$
E_{k}\left(\delta_{1}\left(B_{k k} B_{k k}^{\prime}\right)-\delta_{1}\left(B_{k k}\right) B_{k k}^{\prime}-B_{k k} \delta_{1}\left(B_{k k}^{\prime}\right)\right) E_{s}=0
$$

for $t=2, \ldots, k-1$ and $s=k+1, \ldots, n$. In addition, by (28), it is clear that

$$
\begin{aligned}
& \delta_{1}\left(B_{k k} B_{k k}^{\prime}\right)-\delta_{1}\left(B_{k k}\right) B_{k k}^{\prime}-B_{k k} \delta_{1}\left(B_{k k}^{\prime}\right) \\
& \in \mathscr{T}_{2 k}+\cdots+\mathscr{T}_{k k}+\mathscr{T}_{k(k+1)}+\cdots+\mathscr{T}_{k n} .
\end{aligned}
$$

Now, combining (32)-(34) and (24) gives $\delta_{1}\left(B_{k k} B_{k k}^{\prime}\right)=\delta_{1}\left(B_{k k}\right) B_{k k}^{\prime}+B_{k k} \delta_{1}\left(B_{k k}^{\prime}\right)$.

Step 9.3.4. For any $B_{k k}, B_{k t}^{\prime}$ with $k<t$, we have $\delta_{1}\left(B_{k k} B_{k t}^{\prime}\right)=\delta_{1}\left(B_{k k}\right) B_{k t}^{\prime}+B_{k k} \delta_{1}\left(B_{k t}^{\prime}\right)$.

By (28) and the fact $\delta_{1}\left(B_{k t}\right) \subseteq \mathscr{M}_{k} \cap \mathscr{B}_{1}$, we have

$\delta_{1}\left(B_{k k} B_{k t}^{\prime}\right)=\delta_{1}\left(B_{k k} \circ B_{k t}^{\prime}\right)=\delta_{1}\left(B_{k k}\right) \circ B_{k t}^{\prime}+B_{k k} \circ \delta_{1}\left(B_{k t}^{\prime}\right)$ $=\delta_{1}\left(B_{k k}\right) B_{k t}^{\prime}+B_{k t}^{\prime} \delta_{1}\left(B_{k k}\right)+B_{k k} \delta_{1}\left(B_{k t}^{\prime}\right)+\delta_{1}\left(B_{k t}^{\prime}\right) B_{k k}$ $=\delta_{1}\left(B_{k k}\right) B_{k t}^{\prime}+B_{k k} \delta_{1}\left(B_{k t}^{\prime}\right)$. 
A similar argument to that of Step 9.3.4 can give the following two steps.

Step 9.3.5. For any $B_{k l}, B_{l l}^{\prime}$ with $k<l$, we have $\delta_{1}\left(B_{k l} B_{l l}^{\prime}\right)=\delta_{1}\left(B_{k l}\right) B_{l l}^{\prime}+B_{k l} \delta_{1}\left(B_{l l}^{\prime}\right)$.

Step 9.3.6. For any $B_{k l}, B_{l t}^{\prime}$ with $k<l<t$, we have $\delta_{1}\left(B_{k l} B_{l t}^{\prime}\right)=\delta_{1}\left(B_{k l}\right) B_{l t}^{\prime}+B_{k l} \delta_{1}\left(B_{l t}^{\prime}\right)$.

Step 9.3.7. For any $B_{k l}, B_{s t}^{\prime}$ with $k \leqslant l, s \leqslant t$ and $l \neq s$, we have $\delta_{1}\left(B_{k l}\right) B_{s t}^{\prime}+B_{k l} \delta_{1}\left(B_{s t}^{\prime}\right)=0$.

In fact, if $k \leqslant l<s \leqslant t$, by Step 9.3.2(i), one has

$$
\begin{aligned}
0 & =\delta_{1}\left(B_{k l} \circ B_{s t}^{\prime}\right)=\delta_{1}\left(B_{k l}\right) \circ B_{s t}^{\prime}+B_{k l} \circ \delta_{1}\left(B_{s t}^{\prime}\right) \\
& =\delta_{1}\left(B_{k l}\right) B_{s t}^{\prime}+B_{s t}^{\prime} \delta_{1}\left(B_{k l}\right)+B_{k l} \delta_{1}\left(B_{s t}^{\prime}\right)+\delta_{1}\left(B_{s t}^{\prime}\right) B_{k l} \\
& =\delta_{1}\left(B_{k l}\right) B_{s t}^{\prime}+B_{k l} \delta_{1}\left(B_{s t}^{\prime}\right) .
\end{aligned}
$$

Similarly, if $k \leqslant s<l \leqslant t, k \leqslant s<t \leqslant l$ or $k<s \leqslant$ $t<l$, one can check that $\delta_{1}\left(B_{k l}\right) B_{s t}^{\prime}+B_{k l} \delta_{1}\left(B_{s t}^{\prime}\right)=0$.

Next, if $k=s=t<l$, we have

$$
\begin{aligned}
& \delta_{1}\left(B_{k k}^{\prime} B_{k l}\right)=\delta_{1}\left(B_{k l} \circ B_{k k}^{\prime}\right) \\
& =\delta_{1}\left(B_{k l}\right) B_{k k}^{\prime}+B_{k k}^{\prime} \delta_{1}\left(B_{k l}\right)+B_{k l} \delta_{1}\left(B_{k k}^{\prime}\right)+\delta_{1}\left(B_{k k}^{\prime}\right) B_{k l},
\end{aligned}
$$

and Step 9.3.4 gives $\delta_{1}\left(B_{k l}\right) B_{k k}^{\prime}+B_{k l} \delta_{1}\left(B_{k k}^{\prime}\right)=0$. Similarly, if $s \leqslant k$ and $l \neq s$, by considering subcases $s \leqslant k \leqslant t \leqslant l, s \leqslant k \leqslant l \leqslant t$, and $s \leqslant t \leqslant k \leqslant l$, respectively, one can show that $\delta_{1}\left(B_{k l}\right) B_{s t}^{\prime}+B_{k l} \delta_{1}\left(B_{s t}^{\prime}\right)=0$. The substep is true.

Now, combining Steps 9.3.1-9.3.7, and by a bald calculation, one can show that (25) holds, completing the proof of Step 9.3. It follows from Claim 8 and Steps 9.1-9.3 that $\delta_{1}$ is a derivation.

Claim 10. $\delta$ is an additive derivation on $\mathscr{T}$.

By (1), $\delta=\delta_{1}-d_{1}$ with $d_{1}$ an additive derivation. Now, it follows from Claims 8-9 that $\delta$ is an additive derivation on $\mathscr{T}$.
The proof of Theorem 1 is finished.

Acknowledgements: This work is partially supported by National Natural Science Foundation of China (11671006) and Fund Program for the Scientific Activities of Selected Returned Overseas Professionals in Shanxi Province (20200011).

\section{REFERENCES}

1. Benkovic D (2005) Jordan derivations and antiderivations on triangular matrices. Linear Algebra Appl 397, 235-244.

2. Du YQ, Wang Y (2012) Additivity of Jordan maps on triangular algebras. Linear Multilinear Algebra 60, 933-940.

3. Ji PS, Lai YX, Hou ER (2010) Multiplicative Jordan derivations on Jordan algebras. Acta Math Sin 53, 571-578.

4. Li J, Lu FY (2007) Additive Jordan derivations of reflexive algebras. J Math Anal Appl 329, 102-111.

5. Lu FY (2010) Jordan derivable maps of prime rings. Comm Algebra 38, 4430-4440.

6. Xiao ZK (2012) Nonlinear Jordan derivations of triangular algebras. arXiv:1202.4636v1 [math.RA].

7. Zhang JH, Yu WY (2006) Jordan derivations of triangular algebras. Linear Algebra Appl 419, 251-255.

8. Cheung WS (2001) Commuting maps of triangular algebras. J London Math Soc 63, 117-127.

9. Ferreira BLM (2014) Multiplicative maps on triangular n-matrix rings. Int $J$ Math Game Theory Algebra 23, 1-14.

10. Chen ZH, Hou JC (2001) Multiplicative Lie derivation of triangular 3-matrix rings. arXiv:2001.00427v1 [math.RA].

11. Ferreira BLM, Jr HG (2019) Lie $n$-multiplicative mapping on triangular $n$-matrix rings. Rev Union Mat Argent 60, 9-20.

12. Chen HM, Qi XF (2020) Multiplicative Lie derivations on triangular $n$-matrix rings. Linear Multilinear Algebra. (in press) 